Ann. Biol. anim. Bioch. Biophys., 1979, 19 (2 B), 489-496.

\title{
A. Recherches anatomopathologiques chez le rat ingérant différentes doses d'huile d'arachide ou d'huile de colza à faible teneur en acide érucique (Huile de colza Primor).
}

\section{Etude anatomique générale du myocarde}

\author{
par O. SCHMITT \\ avec la collaboration technique de Thérèse DEGAS, Marie-Reine LANGLOIS et P. PEROT. \\ Loboratoire de Recherches sur la Viande, \\ I.N.R.A., 78350 jouy en Josas.
}

Summary. A. Anatomo-pathological research on rats ingesting different doses of peanut oil or of low-erucic acid rapeseed oil (Primor rapeseed oil). 4. General anatomy of the myocardium.

The general anatomy of the myocardium (histiocytic infiltrations, fibrosis, loose connective tissue, white spots) was studied on 4 rats from each lot. No dose or oil-effect was found. The length of the diet generally tended to influence the development of lesions.

Les conditions expérimentales ont été décrites précédemment. Nous n'insisterons que sur la méthodologie en raison de son caractère d'originaliłé.

\section{Matériel et méthodes.}

Le lendemain de leur arrivée au laboratoire, après la pesée, les animaux ont été anesthésiés par voie intrapéritonéale, lot par lot (4 par 4), au pentobarbital sodique hépariné, puis perfusés selon la méthode de l'eau physiologique héparinée. Après cette perfusion, le système restant en place, l'arrêt du cœur a été provoqué par une piqû re à la xylocaïne à 2 p. 100. Tous les procédés de fixation (Schmitt et Dumont, 1969), jusqu'au moment de l'imprégnation par la paraffine, ont été réalisés par perfusion totale de l'animal à une pression de 0,2 bar (durée $1 \mathrm{~h}$ ). Ont été injectés, par animal :

- le liquide de fixation, 1/2 I (1 vol. chloroforme, 1 vol. alcool absolu, 1/2 vol. acide acétique cristallisable) ;

- le liquide de lavage, 1/2 I (alcool absolu) ;

- l'agent intermédiaire (solvant), 1/2 I (alcool butylique pur).

Chaque cœur, une fois prélevé, a été soumis à une imprégnation par la cyłoparaffine à $56^{\circ} \mathrm{C}$ dans trois bains successifs pendant une journée entière. Toutefois, 

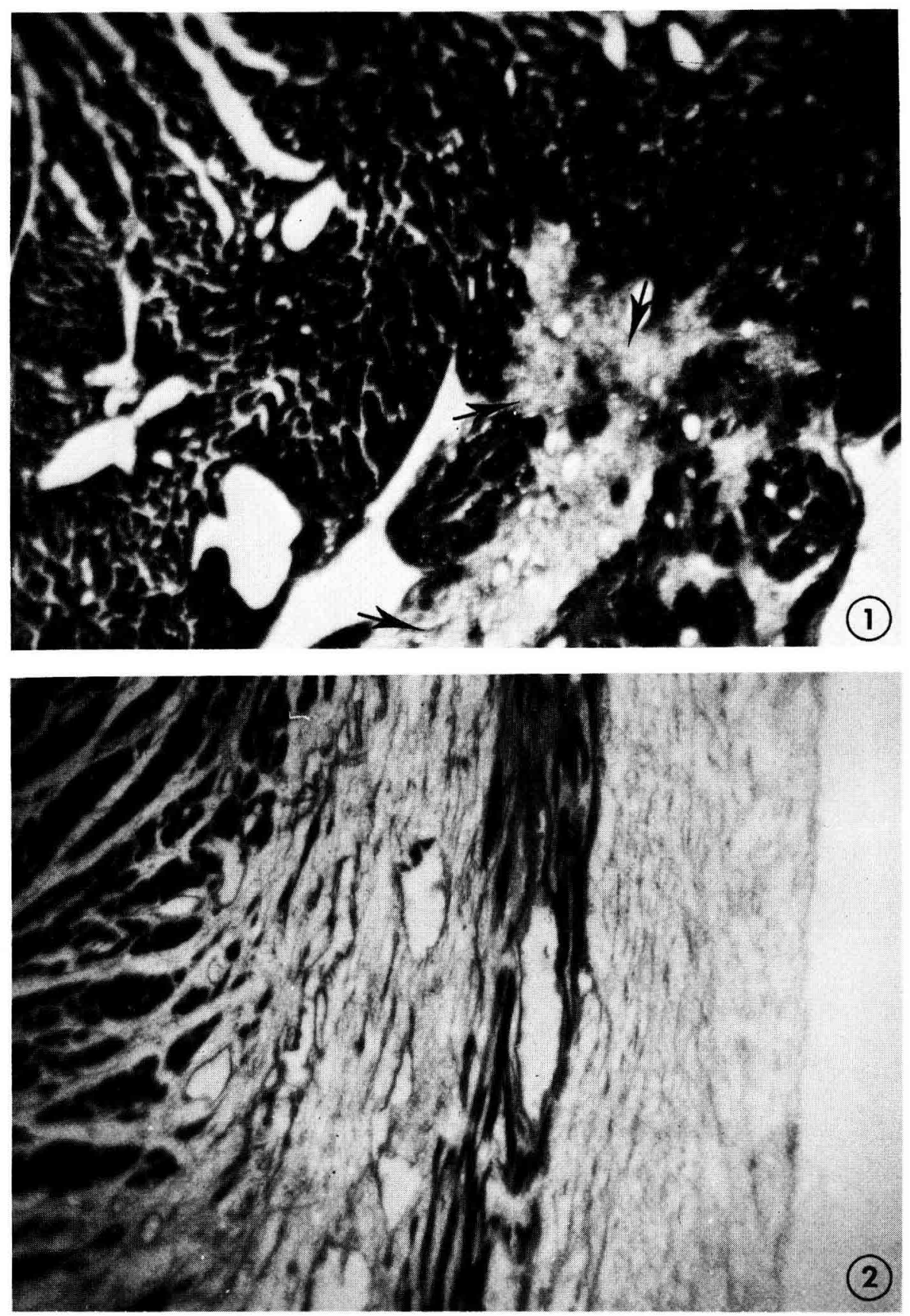

FIG. 1. - Histiocytes ef macrophages $\times 150$.

FIG. 2. - Fibrose en couronne entre du conjonctif très lâche $\times 150$. 
le troisième bain a été composé d'un mélange 50/50 de cyto-paraffine et de paraplast à $56^{\circ} \mathrm{C}$.

Après inclusion, les cœurs ont été débités en série, par coupes de $7 \mu$ d’épaisseur de la pointe du cœur jusqu'à épuisement du muscle. Trois coupes toutes les 50 ont été gardées c'est-à-dire les coupes 1, 2, 3 formant une lame, puis 49, 50, 51 une autre, $99,100,101$ une autre, etc... Ces lames ont été ensuite colorées par le trichrome en un temps selon la méthode de Gabe et Martoja (1968). Pour montrer qu'il n'y a pas de variations dues au colorant d'une lame à l'autre (durée de coloration, épuisement des bains ou température des bains, etc...) les lames $n^{\circ} 10,14$ et 20 de tous les animaux ont été colorées ensemble. Après observation de ces lames témoins, les colorations ont été pratiquées normalement, douze lames par douze lames, animal par animal.

Les lésions diverses (Lenègre, 1957) ont été appréciées par des jugements subjectifs au microscope optique. Les observations aux agrandissements $\times 50$ ef $\times 100$ (oculaire $\times 10$, objectifs $\times 5$ et $\times 10$ ) nous ont permis de juger, subjectivement (j.s.), l'importance des histiocytes et macrophages (fig. 1), la présence de fibroses (fig. 2), l'aspect du tissu conjonctif intersticiel (fig. 3), la présence d'anomalies (taches blanches) dans le tissu conjonctif (fig. 4) et la couleur des préparations. L'échelle subjective suivante a été employée : $0=$ rien, $X=$ présence faible, $X X=$ présence nette, $\mathrm{XXX}=$ présence importante.

\section{Résultats.}

1. Taille des cours.

Une lame (3 coupes) ayant été prélevée toutes les 50 coupes, le nombre de lames obtenues donne une représentation de la longueur du cœur. Ces résultats sont exposés dans le tableau 1.

TABLEAU 1

Nombre de lames obtenues par animal (4 animaux par lot)

\begin{tabular}{|c|c|c|c|c|c|c|c|}
\hline \multicolumn{6}{|c|}{6 mois } & \multicolumn{2}{|c|}{1 an } \\
\hline $\begin{array}{c}5 \text { p. } 100 \mathrm{~A} \\
\left({ }^{1}\right)\end{array}$ & $\begin{array}{l}5 \text { p. } 100 \mathrm{P} \\
\text { (1) }\end{array}$ & $10 \mathrm{P} ._{\mathrm{A}} 100$ & $10 \underset{P}{P_{P}} 100$ & $15 \mathrm{P}_{\mathrm{A}} 100$ & $15 p_{p} 100$ & $\underset{A}{5 \text { p. } 100}$ & 5 P. 100 \\
\hline $\begin{array}{l}37 \\
41 \\
32 \\
51\end{array}$ & $\begin{array}{l}24 \\
50 \\
34 \\
28\end{array}$ & $\begin{array}{l}34 \\
39 \\
32 \\
30\end{array}$ & $\begin{array}{c}3^{*} \\
39^{*} \\
3^{*} \\
34\end{array}$ & $\begin{array}{l}33 \\
33 \\
36 \\
34\end{array}$ & $\begin{array}{l}31 \\
45 \\
28 \\
45\end{array}$ & $\begin{array}{l}35 \\
42 \\
39 \\
43\end{array}$ & $\begin{array}{l}39 \\
39 \\
48 \\
41\end{array}$ \\
\hline
\end{tabular}

* Nous n'avons pu utiliser que trois coupes pour ces deux animaux.

(i) $A=$ Arachide, $P=$ Colza Primor.

2. Examen des cœurs chez les animaux après 6 mois de régime.

Le tableau 2 présente, pour chaque animal, le nombre de lésions pour chaque type de lésion observé (infiltration histiocytaire, fibrose, conjonctif lâche, taches 

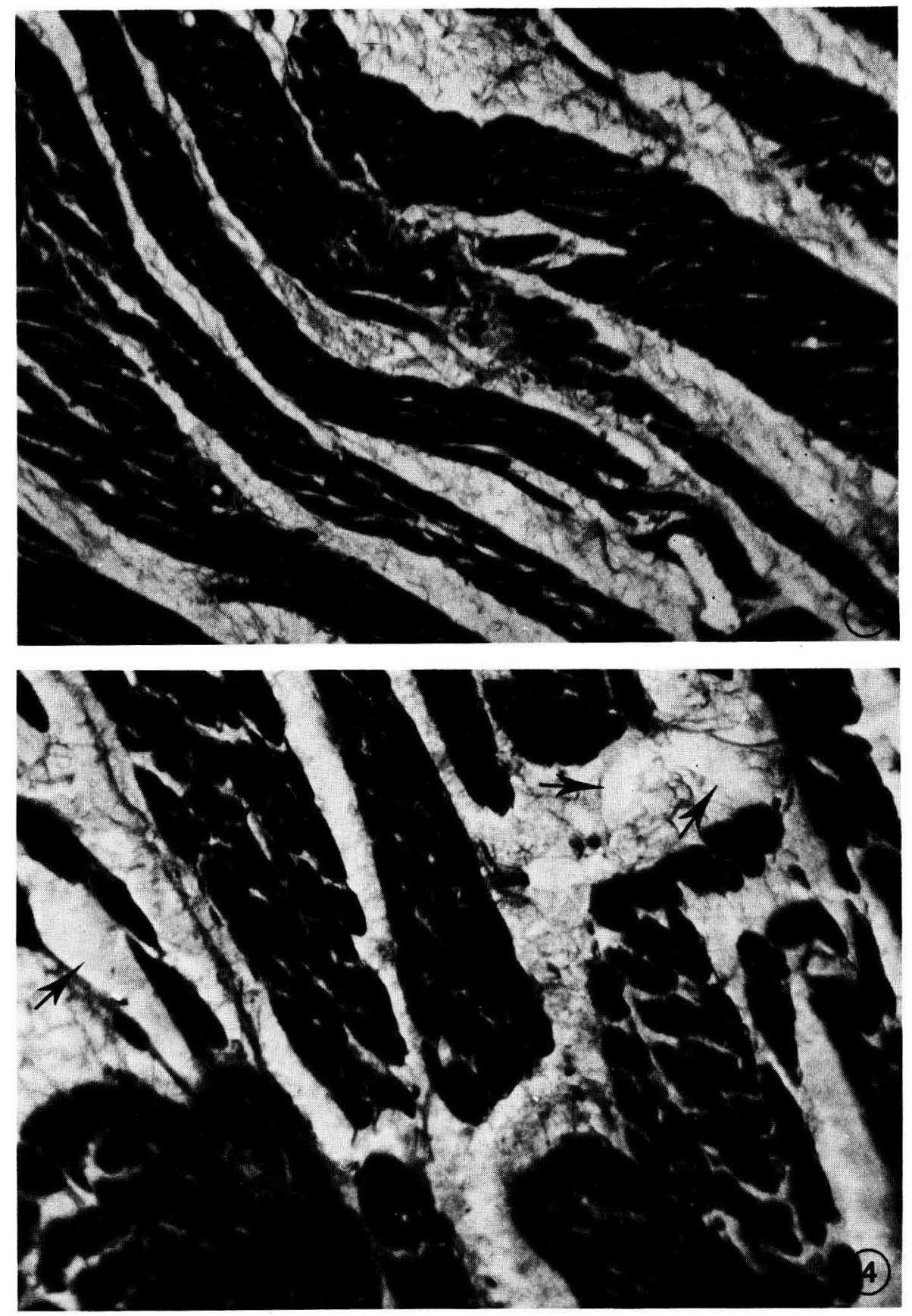

FIG. 3. - Tissu conjonctif lâche $\times 300$.

FIG. 4. - "Taches blanches » dans le tissu conjonctif lâche $\times 300$. 
blanches). On peut aussi, à partir de ce tableau, calculer à quelques microns près la profondeur de la lésion considérée, puisque les coupes sont effectuées tous les $7 \mu$ et qu'une lame est prélevée toutes les 50 coupes.

TABLEAU 2

Examen des cours après 6 mois de régime (4 animaux par régime)

\begin{tabular}{|c|c|c|c|c|c|c|c|c|c|c|c|c|c|c|c|c|}
\hline \multirow{4}{*}{ Régime } & \multicolumn{8}{|c|}{ Arachide } & \multicolumn{8}{|c|}{ Colza Primor } \\
\hline & \multirow{2}{*}{\multicolumn{2}{|c|}{$\begin{array}{l}\text { Histio- } \\
\text { cyte }\end{array}$}} & \multirow{2}{*}{\multicolumn{2}{|c|}{ Fibrose }} & \multicolumn{4}{|c|}{ Conjonctif } & \multirow{2}{*}{\multicolumn{2}{|c|}{ Histiocyfe }} & \multirow{2}{*}{\multicolumn{2}{|c|}{ Fibrose }} & \multicolumn{4}{|c|}{ Conjonctif } \\
\hline & & & & & \multicolumn{2}{|c|}{ lâche } & \multicolumn{2}{|c|}{ blanc } & & & & & \multicolumn{2}{|c|}{ lâche } & \multicolumn{2}{|c|}{ blanc } \\
\hline & $\mathbf{L}$ & G & $\mathbf{L}$ & $\mathbf{G}$ & $\mathbf{L}$ & G & $\mathbf{L}$ & G & $\mathbf{L}$ & $\mathbf{G}$ & $\mathbf{L}$ & G & $\mathbf{L}$ & $\mathbf{G}$ & $\mathbf{L}$ & $\mathbf{G}$ \\
\hline \multirow{4}{*}{5 p. 100} & 3 & $x$ & 3 & $x$ & 14 & $x$ & 14 & $x$ & 0 & 0 & 0 & 0 & 0 & 0 & 0 & 0 \\
\hline & 8 & $x$ & 0 & 0 & 5 & $x$ & 0 & 0 & 0 & 0 & 0 & 0 & 49 & $\times \times \times$ & 49 & $x \times x$ \\
\hline & 2 & $x$ & 0 & 0 & 0 & 0 & 0 & 0 & 0 & 0 & 0 & 0 & 0 & 0 & 0 & 0 \\
\hline & 19 & $\times x$ & 9 & 0 & 14 & $x$ & 26 & $x$ & 0 & 0 & 0 & 0 & 25 & $\times \times \times$ & 25 & $x \times x$ \\
\hline \multirow{4}{*}{10 p. 100} & 0 & 0 & 0 & 0 & 5 & $\times$ & 0 & 0 & 0 & 0 & 0 & 0 & 0 & 0 & 0 & 0 \\
\hline & 0 & 0 & 0 & 0 & 39 & $x \times$ & 0 & 0 & 24 & $x \times x$ & 0 & 0 & 28 & $x$ & 28 & $x$ \\
\hline & $\overline{0}$ & 0 & 0 & $\overline{0}$ & 23 & $x$ & 0 & 0 & 0 & 0 & 0 & 0 & 0 & 0 & 0 & 0 \\
\hline & 0 & 0 & 0 & 0 & 0 & 0 & 0 & 0 & 10 & $x \times$ & 10 & + & 28 & $x$ & 16 & $x$ \\
\hline \multirow{4}{*}{15 p. 100} & 0 & 0 & 0 & 0 & 0 & 0 & 0 & 0 & 15 & $\times \times$ & 15 & $x$ & 0 & 0 & 19 & $x$ \\
\hline & 0 & 0 & 0 & 0 & 0 & 0 & 0 & 0 & 15 & $x \times$ & 19 & $\times x$ & 40 & $x$ & 42 & $x$ \\
\hline & $\overline{20} \mid$ & $x \times x$ & $\overline{0}$ & $\overline{0}$ & 0 & 0 & 0 & 0 & 0 & 0 & 0 & 0 & 0 & 0 & 0 & $x$ \\
\hline & 8 & $\times \times x$ & 0 & 0 & 0 & 0 & 0 & 0 & 0 & 0 & 0 & $x \times \mid$ & 45 & $x$ & 45 & $x$ \\
\hline
\end{tabular}

$L=$ nombre de lames, $G=$ gravité des lésions.

Infiltration histiocytaire. - Cinq animaux sur onze dans le cas de l'arachide et six animaux sur dix dans le cas du colza Primor sont exempts de ce type de lésion. A 5 p. 100 d'huile dans le régime, seul le lot arachide présente des lésions faibles ou netfes. A 15 p. 100 , deux animaux sur quatre dans les deux lots sont indemnes de lésions. Les deux autres présentent des lésions sévères plus marquées dans le lot arachide.

Fibrose. - Un seul animal sur onze du lot arachide et trois animaux sur dix du lot colza Primor présentent des fibroses légères. Un animal du lot colza Primor présente une fibrose nette. A 15 p. 100 d'huile dans le régime, tous les animaux du lot 
arachide ef un seul du lot colza Primor sont indemnes de lésions diverses. Il en est de même à 10 p. 100 d'huile dans le régime. A 5 p. 100 d'huile, un seul animal (arachide) présente une légère fibrose.

Conjonctif lâche et taches blanches. - Cinq animaux sur onze dans le cas de l'arachide et trois animaux sur dix dans le cas du colza Primor sont indemnes de ce type de lésion. II n'y a pas apparemment d'effet « dose », puisqu'on trouve ces lésions dans le lot 5 p. 100 d'arachide et qu'on n'en trouve pas dans le lot à 15 p. 100 et que, dans le cas du colza Primor, les lésions du lot à 5 p. 100 sont plus sévères que celles du lot à 15 p. 100. Si l'on ne tient compte que des « taches blanches », le lot colza Primor est plus afteint que le lot arachide.

3. Examen des cœurs chez les animaux soumis à 1 an de régime.

Seul « l'effet nature de l'huile » peut être analysé. Les résultats sont consignés dans le tableau 3.

TABLEAU 3

Examen des cœurs après 1 an de régime à 5 p. 100 (4 animaux par régime)

\begin{tabular}{|c|c|c|c|c|c|c|c|c|c|c|c|c|c|c|c|}
\hline \multicolumn{8}{|c|}{ Arachide } & \multicolumn{8}{|c|}{ Colza Primor } \\
\hline \multicolumn{2}{|c|}{ Histiocyte } & \multicolumn{2}{|c|}{ Fibrose } & \multicolumn{2}{|c|}{$\begin{array}{l}\text { Conjonctif } \\
\text { lâche }\end{array}$} & \multicolumn{2}{|c|}{$\begin{array}{c}\text { Conjonctif } \\
\text { blane }\end{array}$} & \multicolumn{2}{|c|}{ Histiocyte } & \multicolumn{2}{|c|}{ Fibrose } & \multicolumn{2}{|c|}{$\begin{array}{c}\text { Conjonctif } \\
\text { lâche }\end{array}$} & \multicolumn{2}{|c|}{$\begin{array}{c}\text { Conjonctif } \\
\text { blanc }\end{array}$} \\
\hline $\mathbf{P}$ & G & $\mathbf{P}$ & G & $P$ & G & $P$ & G & $\mathbf{P}$ & G & $\mathbf{P}$ & G & $\mathbf{P}$ & G & $\mathbf{P}$ & G \\
\hline $\begin{array}{r}14350 \\
5600 \\
0 \\
13650\end{array}$ & $\begin{array}{c}\times x \times \\
\times \\
0 \\
\times \times\end{array}$ & $\begin{array}{r}14340 \\
0 \\
0 \\
13650\end{array}$ & $\mid \begin{array}{c}\times \times \times \\
0 \\
0 \\
\times \times \times\end{array}$ & $\begin{array}{l}0 \\
0 \\
0 \\
0\end{array}$ & $\begin{array}{l}0 \\
0 \\
0 \\
0\end{array}$ & $\begin{array}{l}0 \\
0 \\
0 \\
0\end{array}$ & $\begin{array}{l}0 \\
0 \\
0 \\
0\end{array}$ & $\begin{array}{r}700 \\
12250 \\
0 \\
0\end{array}$ & $\begin{array}{c}\times \\
\times \times \times \\
0 \\
0\end{array}$ & $\begin{array}{l}0 \\
0 \\
0 \\
0\end{array}$ & $\begin{array}{l}0 \\
0 \\
0 \\
0\end{array}$ & $\left|\begin{array}{r}12950 \\
9450 \\
14000 \\
0\end{array}\right|$ & $\begin{array}{c}x \\
x \\
x \\
0\end{array}$ & $\begin{array}{r}12950 \\
7700 \\
14000 \\
0\end{array}$ & $\begin{array}{l}x \\
x \\
x \\
0\end{array}$ \\
\hline
\end{tabular}

$P=$ profondeur de la lésion en microns, $G=$ gravité des lésions.

Infiltration histiocytaire. - Un animal sur quatre dans le lot arachide ef deux animaux sur quatre dans le lot colza Primor sont indemnes de lésions ; parmi les animaux atteints, deux le sont sévèrement (profondeur dans chacune des lésions : $14 \mathrm{~mm}$ ) dans le cas de l'arachide ef un presque aussi sévèrement dans le cas du colza Primor.

Fibrose. - Aucune fibrose n'a été décelée dans le lot colza Primor, alors que deux animaux sur quatre ont été sérieusement atteints dans le cas du lot arachide.

Conjonctif lâche et taches blanches. - Les cours des animaux du lot arachide ne sont pas affectés, alors que trois animaux sur quatre du lot colza Primor sont atteints par cette sorte d'anomalie du conjonctif. Toutefois, la gravité de ces lésions reste faible. 


\section{Discussion et conclusion.}

Il est difficile, compte tenu du petit nombre d'animaux, de donner à nos résultats une signification statistique. En revanche, la technique utilisée, par l'examen systématique d'une lame toutes les 50 coupes, permet d'analyser avec précision la « profondeur » des lésions pour un cœur donné.

La seule constatation indiscutable est la grande variabilité des résultats. Dans un lot donné, on trouve la plupart du temps des animaux sains et des animaux plus ou moins gravement atteints. L'effet « dose » ne se dégage pas avec évidence de nos résultats : si l'on ne tient compte que des infiltrations histiocytaires et des fibroses, seuls les lots arachide à 10 p. 100 et colza Primor à 5 p. 100 sont exempts de lésions.

Dans le cas de l'arachide, le lot à 15 p. 100 d'huile ne présente pas de fibrose ; mais les deux animaux atteints d'infiltrations histiocytaires le sont gravement. Cependant, tous les animaux du lot à 5 p. 100 d'huile sont atteints d'infiltrations histiocytaires légères et un seul cœur souffre de fibrose.

Dans le cas du colza Primor, ce sont les animaux du lot à 10 p. 100 qui possèdent le plus d'histiocytes, mais ce sont ceux du lot à 15 p. 100 qui sont le plus atteints par des fibroses. En revanche, ce sont les animaux à 5 p. 100 qui présentent le plus d'anomalies du tissu conjonctif.

La comparaison des « huiles » après six mois de régime permet de dégager les tendances suivantes:

- à 5 p. 100 dans le régime, les infiltrations histiocytaires et les fibroses existent dans le lot arachide, alors qu'elles sont absentes dans le lot colza Primor. Pour ce dernier, on constate cependant davantage d'anomalies du tissu conjonctif ;

- à 10 p. 100 d'huile, seul le lot colza Primor est atteint par des histiocytes et des macrophages, ainsi qu'une légère fibrose. Les anomalies du tissu conjonctif sont comparables dans les deux lots ;

- à 15 p. 100 d'huile, les infiltrations histiocytaires sont plus sévères dans le loł arachide, alors que seul le lot colza Primor présente des fibroses ( 3 animaux sur quatre) ;

Après un an de régime et à 5 p. 100 d'huile, les animaux colza Primor ne présentent pas de fibrose, alors que deux animaux sur quatre du régime " arachide » son sévèrement atteints. Pour ce qui est des histiocytes et des macrophages, deux animaux sont gravement atteints ef un moyennement dans le lot arachide, alors qu'un seul animal l'est sévèrement ef un autre moyennement dans le lot colza Primor. En revanche, d'autres anomalies du tissu conjonctif ne se trouvent que chez les animaux du lot colza Primor.

Pour une huile donnée, quel est l'effet de la durée de l'expérience ? II semblerait qu'il y ait une tendance à un développement des lésions au cours du temps. On trouve toujours des animaux sains à un an (deux dans le lot colza Primor, un dans le lot arachide, si l'on ne tient pas compte des autres anomalies du conjonctif) mais des infiltrations histiocyłaires apparaissent dans le lot colza Primor, alors qu'elles étaient absentes à six mois et celles du lot arachide se sont aggravées. Les fibroses sont plus importantes à un an qu'à six mois dans le lot arachide ; elles sont toujours absentes dans le lot colza Primor. Par contre, le lot colza Primor défient d'autres anomalies du tissu conjonctif et elles subsistent encore à un an. 
Des conclusions plus nettes auraient peut-être éventuellement été dégagées de ce travail, si nous avions travaillé sur un plus grand nombre d'animaux. Elles auraient sans doute confirmé les tendances qui se dégagent de cette discussion. D'une manière globale et schématique, faute d'une analyse statistique détaillée et d'une interprétation physiologique approfondie, on peut dire que nous n'avons mis en évidence ni un effet dose, ni un effet « huile ». Il semblerait, toutefois, qu'une tendance se dessine pour l'effet « durée du régime alimentaire »: les lésions rencontrées chez les animaux des deux régimes seraient plus graves à un an qu'à six mois.

Effets physiologiques comparés des huiles de colza et d'arachide. ATP-INRA, 1974-1976.

Remerciements. - Nous remercions MM. Pointillart et Delpal de leurs conseils avertis pour mener à bien ce travail.

\section{Références}

GABE M., MARTOJA M., 1968, in GABE M., Techniques histologiques. Masson et Cie, édit. Paris. LENEGRE J., 1957. Archives des maladies du cœur ef des vaisseaux. 50e année, suppl. 1.

SCHMITT O., DUMONT B. L., 1969. Méthode d'analyse de la structure musculaire. Ann. Biol. anim. Bioch. Biophys., 9, 123-134. 